\title{
Quantum tunneling of two coupled single-molecular magnets
}

\author{
Jian-Ming Hu, ${ }^{1}$ Zhi-De Chen, ${ }^{2}$ and Shun-Qing Shen ${ }^{1}$ \\ ${ }^{1}$ Department of Physics, The University of Hong Kong, Pokfulam Road, Hong Kong, China \\ ${ }^{2}$ Department of Physics and Institute of Modern Condensed Matter Physics, Guangzhou University, Guangzhou 510405, China
}

(Received 11 November 2002; revised manuscript received 21 May 2003; published 8 September 2003)

\begin{abstract}
Two single-molecule magnets are coupled antiferromagnetically to form a supramolecule dimer. We study the coupling effect and tunneling process by means of the numerical exact diagonalization method, and apply them to the recently synthesized supramoleculer dimer $\left[\mathrm{Mn}_{4}\right]_{2}$. A stray field is introduced to explain the tunneling splitting. Based on the Landau-Zener model the present theory is in qualitative agreement with the sweeping rate effect on the step height in the hysteresis loops.
\end{abstract}

DOI: 10.1103/PhysRevB.68.104407

PACS number(s): 75.45.+j, 75.50.Xx

Nanometer-sized magnetic particles and clusters have generated continuous interest as study of their properties has proved to be scientifically and technologically very challenging. ${ }^{1-7}$ Up to now magnetic molecular clusters have been one of the most promising candidates for observing macroscopic quantum phenomena since they have a welldefined structure with a well-characterized spin ground state and anisotropy. ${ }^{8}$ One of the well-studied systems is the single-molecule magnet $(\mathrm{SMM}) \mathrm{Mn}_{4} \cdot{ }^{9-12}$ The molecule has a well-isolated ground state with a half integer spin $S=9 / 2$, and magnetization tunneling is observed at zero magnetic field. Very recently a supramolecular dimer of two SMMs $\left[\mathrm{Mn}_{4} \mathrm{O}_{3} \mathrm{Cl}_{4}\left(\mathrm{O}_{2} \mathrm{CEt}\right)_{3}(p y)_{3}\right]_{2}\left(\left[\mathrm{Mn}_{4}\right]_{2}\right)$ was reported to be synthesized successfully by Wernsdorfer, Allaga-Alcalde, Hendrickson, and Christou (WAHC). ${ }^{13}$ The antiferromagnetic coupling between two SMMs leads to this dimer with a spin singlet ground state and makes the quantum tunneling quite different from SMMs $\mathrm{Mn}_{4}$. The coupling also makes this dimer an excellent candidate for studying quantum tunneling in a system of two truly and coupled identical particles. Quantum tunneling of magnetization can be an advantage for some applications of SMMs in providing quantum superpositions of states required for quantum computing. ${ }^{14,15}$ So the coupling effect in quantum tunneling between SMMs is a very important issue for the application of integrated molecular magnets. In this paper we first study the tunneling process in one $\mathrm{SMM} \mathrm{Mn}_{4}$ with spin $S=9 / 2$. A local stray field has to be introduced to explain the tunneling of the ground state at zero field. ${ }^{12}$ Then we study the coupling effect of two SMMs and observe the effect that the coupling provides a bias field to split the tunneling points into two separated ones, and the tunneling splitting at the original point is suppressed. We apply our observation to the newly synthesized supramolecular dimer of two SMMs $\left[\mathrm{Mn}_{4}\right]_{2}$. Finally, we explain the sweeping rate effect in the derivatives of hysteresis loops based on the Landau-Zener model.

We first start with a biaxial model for a SMM $\mathrm{Mn}_{4}$ with spin $S=9 / 2$,

$$
H_{i}=-D \mathbf{S}_{z i}^{2}+E\left(\mathbf{S}_{x i}^{2}-\mathbf{S}_{y i}^{2}\right)+g \mu_{B} \mu_{0} \mathbf{S}_{i} \cdot(\mathbf{B}+\mathbf{h}),
$$

where $i=1$ or 2 referring to the two SMMs in the dimer, $D$ and $E$ are the axial anisotropic constants, and $\mathbf{B}=B e_{z}$ is the external magnetic field along the $z$ axis. The term $g \mu_{B} \mu_{0} \mathbf{S}_{i}$
- $\mathbf{h}$ is the local stray field interaction between the SMMs and the environment. For simplicity we denote the energy eigenstate of the biaxial model $|m\rangle$ by its dominant $S_{z}=m$ component, and $m=-S,-S+1, \cdots, S$. The $E$ term and the stray field may lead to some minor corrections to these states. If the stray field is not included, it is well known that for a half integer spin the tunneling between the states $|-S\rangle$ and $|S\rangle$ is quenched due to the parity symmetry. ${ }^{16-18}$ It can be proved simply that, for any integer $n$, we always have $\left\langle-S\left|\left(H_{i}\right)^{n}\right| M\right\rangle=0 \quad$ with $M=-S+1,-S+3, \cdots, S$. The equality indicates that there is no connection or that no tunneling occurs between these states $|-S\rangle$ and $|M\rangle$. Experimentally quantum tunneling was observed in a $\mathrm{SMM} \mathrm{Mn}_{4}$ between the states $|-S\rangle$ and $|S\rangle .{ }^{10}$ So there must be a small internal magnetic field by the nuclear spins of the Mn ions and/or the dipole-dipole interaction between different molecules. We model the interaction as a local stray field $\mathbf{h}$ with a random Gaussian distribution with the equal width $\sigma$ in three directions, as we did for the molecular magnets $\mathrm{Fe}_{8},{ }^{19}$

$$
P(\mathbf{h})=\frac{1}{\left(2 \pi \sigma^{2}\right)^{3 / 2}} \exp \left[-\mathbf{h}^{2} / 2 \sigma^{2}\right] .
$$

The interaction may originate from the dipole-dipole interaction. A transverse component of such a field may lead to a tunneling splitting at zero field as observed in Ref. 10. In this paper we take the parameters for a SMM $\mathrm{Mn}_{4}$ to be $D$ $=0.762 \mathrm{~K}, E=0.0317 \mathrm{~K},{ }^{10,11}$ and $\sigma=0.035 \mathrm{~T}^{12}$ The resulting tunneling splitting for the ground states $|-9 / 2\rangle$ and $|9 / 2\rangle$ at zero field is $\sqrt{\left\langle\Delta_{0}^{2}\right\rangle}=3.280 \times 10^{-7} \mathrm{~K}$, and that for the states $|-9 / 2\rangle$ and $|7 / 2\rangle$ is $\sqrt{\left\langle\Delta_{1}^{2}\right\rangle}=1.52627 \times 10^{-5} \mathrm{~K}$ by using the exact diagonalization method where $\langle\ldots\rangle$ stands for the averaging over the stray field. ${ }^{20}$ Thus the local stray field may cause a tunneling splitting between the ground states.

Following Wernsdorfer et al., the two $\mathrm{SMM} \mathrm{Mn}_{4} \mathrm{~s}$ in the dimer $\left[\mathrm{Mn}_{4}\right]_{2}$ are coupled via a weak antiferromagnetic superexchange coupling $J$. Thus the model Hamiltonian for the dimer is

$$
H=H_{1}+H_{2}+J \mathbf{S}_{1} \cdot \mathbf{S}_{2},
$$

where $S_{1}=S_{2}=9 / 2$. For each dimer there are $\left(2 S_{1}\right.$ $+1)\left(2 S_{2}+1\right)=100$ energy eigenstates. Like in a SMM $\mathrm{Mn}_{4}$, each state can be labeled approximately by two pre- 


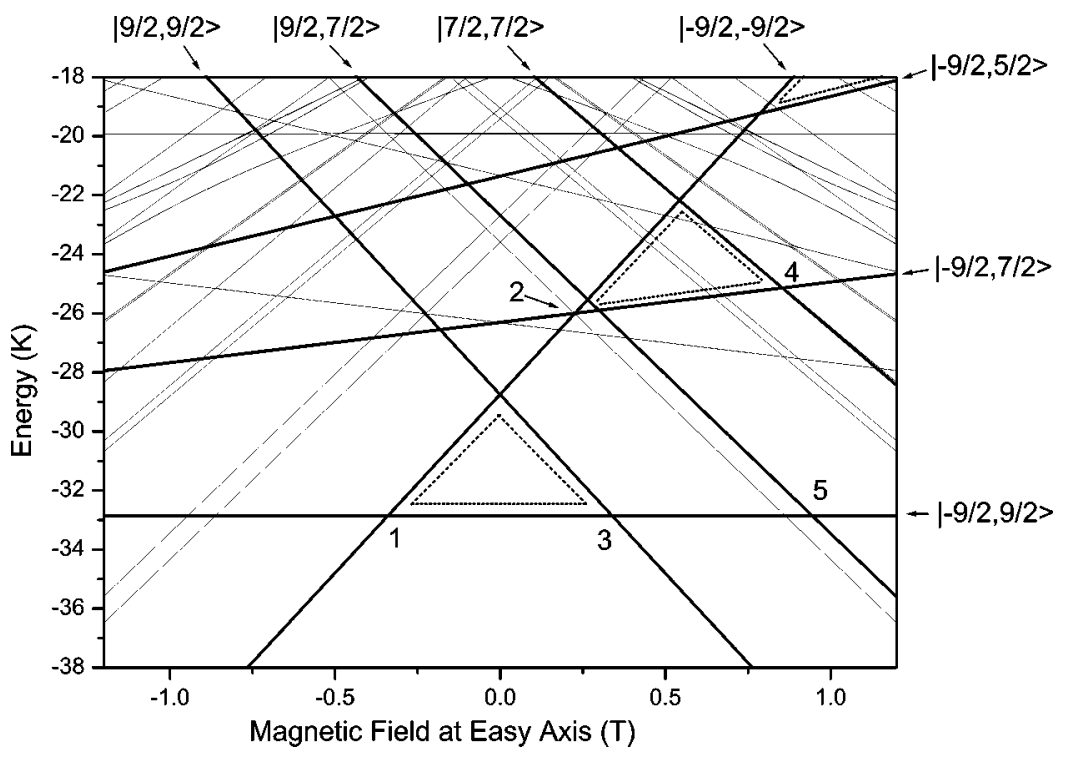

FIG. 1. Spin state energy of the model Hamiltonian [Eq. (1)] for $\left[\mathrm{Mn}_{4}\right]_{2}$ without a local stray field as a function of applied magnetic field by taking $D=0.762 \mathrm{~K}, E=0.0317 \mathrm{~K}$, and $J$ $=0.1 \mathrm{~K}$. The two triangles are related to the tunneling (i) from $|-9 / 2,-9 / 2\rangle_{+}$to $|-9 / 2,9 / 2\rangle_{+}$at point 1 , then to $|9 / 2,9 / 2\rangle_{+}$at point 3 , and (ii) from $|-9 / 2,-9 / 2\rangle_{+}$to $|-9 / 2,7 / 2\rangle_{+}$at point 2 , then to $|7 / 2,7 / 2\rangle_{+}$at point 4 . The point 5 is from $|-9 / 2,9 / 2\rangle_{+}$to $|7 / 2,9 / 2\rangle_{+}$. The resonance fields for the five points are $-0.335 \mathrm{~T}, 0.233 \mathrm{~T}$, $0.335 \mathrm{~T}, 0.861 \mathrm{~T}$, and $0.943 \mathrm{~T}$, respectively.

dominant quantum numbers $\left|m_{1}, m_{2}\right\rangle$ for two SMMs with $m_{1,2}=-9 / 2,-7 / 2, \cdots, 9 / 2$. Without the coupling $J$ the states $\left|m_{1}, m_{2}\right\rangle$ and $\left|m_{2}, m_{1}\right\rangle$ are degenerated. Since the two SMMs can be regarded to be truly identical particles permutation symmetry between particle 1 and 2 exists and the eigenstates may possess parity symmetry. Thus the eigenstates for the system are denoted by $\left|m_{1}, m_{2}\right\rangle_{+}$for even parity and $\left|m_{1}, m_{2}\right\rangle_{-}$for odd parity. The antiferromagnetic coupling $J$ may remove the degeneracy of these two states, but the parity in the states remains unchanged. Even when we take into account the coupling $J$ and the transverse terms the states become a linear combination of all possible states, and for simplicity, we still use the two dominant quantum numbers to represent the states. All the energy eigenvalues that result from neglecting the local stray fields are plotted in Fig. 1. The average over the local stray field does not change the position of the energy-level crossing by much.

Before explaining the experimental observation from the dimer we first consider the effect caused by the coupling $J$ between the two particles. By assuming that the tunneling between the states $\left|m_{1}\right\rangle$ and $\left|m_{1^{\prime}}\right\rangle$ under a sweeping field $\mathbf{B}\left(=-c_{b} t\right)$ and the tunneling splitting between the two states is $\Delta$, the pair of the splitting energy eigenvalues near the resonant point can be written as

$$
\varepsilon_{ \pm}=\frac{1}{2}\left\{\left(m_{1}+m_{1^{\prime}}\right) b \pm \sqrt{\left[\left(m_{1}-m_{1^{\prime}}\right) b\right]^{2}+\Delta^{2}}\right\}
$$

with $b=g \mu_{B} \mu_{0} \hbar B$, and the two states are given by

$$
\phi_{ \pm}^{1}(b)=\left( \pm c_{ \pm}\left|m_{1}\right\rangle+c_{\mp}\left|m_{1^{\prime}}\right\rangle\right) / \sqrt{2}
$$

with

$$
c_{ \pm}=\sqrt{1 \pm\left(m_{1}-m_{1^{\prime}}\right) b / \sqrt{\left[\left(m_{1}-m_{1^{\prime}}\right) b\right]^{2}+\Delta^{2}}} .
$$

Before the resonant tunneling, the initial state is at $\left|m_{1}\right\rangle$, i.e., at $b=+\infty, \phi_{+}(b) \rightarrow\left|m_{1^{\prime}}\right\rangle$, and $\phi_{-}(b) \rightarrow\left|m_{1}\right\rangle$; after the tunneling, it is at $b=-\infty, \phi_{+}(b) \rightarrow\left|m_{1}\right\rangle$, and $\phi_{-}(b)$ $\rightarrow-\left|m_{1^{\prime}}\right\rangle$. Thus $\phi_{+}(b)$ changes from $\left|m_{1^{\prime}}\right\rangle$ to $\left|m_{1}\right\rangle$, while $\phi_{-}(b)$ changes from $\left|m_{1}\right\rangle$ to $-\left|m_{1^{\prime}}\right\rangle$. The energies of the two states are avoided near the resonant point if $\Delta \neq 0$. When two identical particles are put together there are four possible states: $|+,+\rangle_{+}=\phi_{+}^{1} \otimes \phi_{+}^{2} \quad$ with the energy $2 \varepsilon_{+}$, $|+,-\rangle_{ \pm}=\left(\phi_{+}^{1} \otimes \phi_{-}^{2} \pm \phi_{-}^{1} \otimes \phi_{+}^{2}\right) / \sqrt{2}$ with the energy $\varepsilon_{+}$ $+\varepsilon_{-}$, and $|-,-\rangle_{+}=\phi_{-}^{1} \otimes \phi_{-}^{2}$ with the energy $2 \varepsilon_{-}$. We denote the even and odd parities of the states by the subscripts \pm , respectively. The energies vary with the external field, and are plotted in Fig. 2, for an illustration, by choosing $m_{1}=m_{2}=-9 / 2$ and $m_{1^{\prime}}=m_{2^{\prime}}=7 / 2$ for the model in Eq. (3). The tunneling splitting between the two states $|+,+\rangle_{+}$ and $|-,-\rangle_{+}$is $2 \Delta$, the double of a single particle, as expected. To see the coupling effect of two particles, we plot the energy eigenvalues for several different couplings in Fig. 2. It is obtained by the exact diagonalization of the 100 $\times 100$ matrix for the Hamiltonian. The two states $|+,-\rangle_{ \pm}$ are degenerated for $J=0.0$. A small amount of coupling $J$ may remove the degeneracy of the two states. The state $+,-\rangle_{-}$has odd parity and does not take part in the tunneling process since the other three states have even parity. It is shown that the coupling $J$ leads to two consequences: (i) The tunneling splitting from $|-,-\rangle_{+}$and $|+,+\rangle_{+}$decreases very quickly with increasing $J$, and almost closes for $J$ $>0.3 \times 10^{-5} \mathrm{~K}$. In the dimer of $\left[\mathrm{Mn}_{4}\right]_{2}$ the coupling $J \approx 0.1$ $\mathrm{K}$ and tends to suppress the tunneling at this point completely. (ii) The tunneling splitting from $|-,-\rangle_{+}$to $\mid+$, $+\rangle_{+}$occurs at two separated points via an intermediate state $|+,-\rangle_{+}$. The coupling $J$ provides an inner bias field to expel the two resonant points away from the original ones of $|+,+\rangle_{+}$and $|+,-\rangle_{+}$. For convenience of analyzing the tunneling we introduce the "triangle process" to reflect the tunneling structure of the two identical particles. In the language of $m_{1}$ and $m_{2}$, the process from $\left|m_{1}, m_{1}\right\rangle_{+}$to $\left|m_{1^{\prime}}, m_{1^{\prime}}\right\rangle_{+}$is described as follows: the first resonant tunneling occurs from $\left|m_{1}, m_{1}\right\rangle_{+}$to $\left|m_{1}, m_{1^{\prime}}\right\rangle_{+}$, and the second 

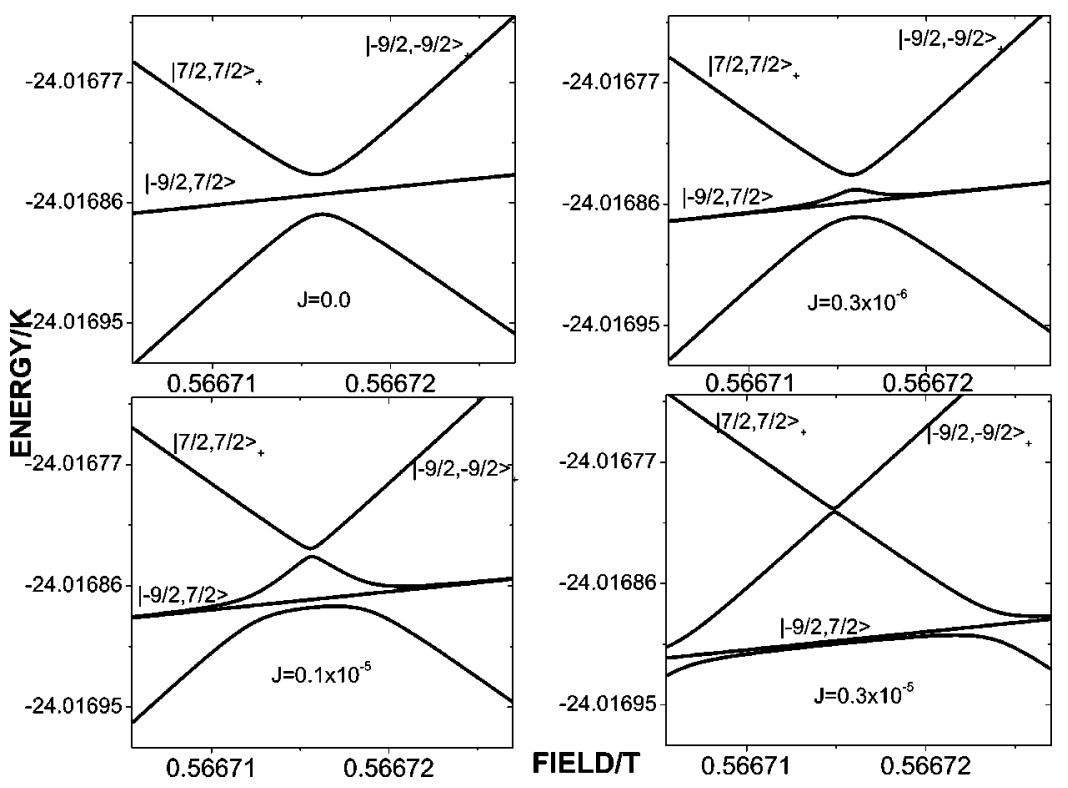

FIG. 2. Energy levels of the states of two coupled identical particles $|-9 / 2,-9 / 2\rangle_{+}$, $|7 / 2,7 / 2\rangle_{+}$, and $|-9 / 2,7 / 2\rangle_{ \pm}$versus the sweeping field at different couplings $J$ as an example to illustrate the coupling effect of the tunneling process from $\left|m_{1}, m_{1}\right\rangle_{+}$to $\mid m_{\left.1^{\prime}, m_{1}\right\rangle_{+}}$via $\left|m_{1}, m_{1}\right\rangle_{+}$( $x$ axis: magnetic field at easy axis/ $T ; y$ axis: energy $\mathrm{K})$. one follows from $\left|m_{1}, m_{1^{\prime}}\right\rangle_{+}$to $\left|m_{1^{\prime}}, m_{1^{\prime}}\right\rangle_{+}$. The explicit tunneling from $\left|m_{1}, m_{1}\right\rangle_{+}$to $\left|m_{1^{\prime}}, m_{1^{\prime}}\right\rangle_{+}$is suppressed completely by the coupling $J$. The three points form a triangle.

Now we are ready to analyze the quantum tunneling in the dimer. Typical hysteresis loops in magnetization versus sweeping external field applied along the easy axis are observed. These loops display steplike features separated by plateaus. The step heights become temperature independent below $0.3 \mathrm{~K}$, but depend on the sweeping rate of magnetic field $c=d B / d t$. Derivatives of the loops at different sweeping rates reflect that quantum tunneling occurs at several points, but is absent at zero field. At high field the initial state is $|-9 / 2,-9 / 2\rangle_{+}$, which has even parity. Due to the permutation symmetry of identical particles all the tunneling to the states $\left|m_{1}, m_{2}\right\rangle_{-}$with odd parity in this system are prohibited. The tunneling process in the dimer can be understood essentially to be two triangle processes as shown in Fig. 1. Starting from the initial state $|-9 / 2,-9 / 2\rangle_{+}$, the first level crossing happens at magnetic field $b_{1}=-0.33 \mathrm{~T}$, which is from $|-9 / 2,-9 / 2\rangle_{+}$to $|-9 / 2,9 / 2\rangle_{+}$at point 1 , and the dual resonant point is from $|-9 / 2,9 / 2\rangle_{+}$to $|9 / 2,9 / 2\rangle_{+}$at point 3 . $b_{3}=+0.33 \mathrm{~T}$ in the first triangle process. The energy of the intermediate state $|-9 / 2,9 / 2\rangle_{+}$is independent of the external field. The resonant field for points 1 and 3 are $b_{1,3}$ $= \pm 9 J / 2 g \mu_{B} \mu_{0}$ from the model [Eq. (3)]. Thus $J$ is calculated to be $0.1 \mathrm{~K}$ as Wernsdorfer et al. found. The finite coupling does not lead to a tunneling splitting at this point, which can be proved explicitly: for an integer $n$ we have

$$
{ }_{+}\left\langle-9 / 2,-9 / 2\left|H^{n}\right|-9 / 2,9 / 2\right\rangle_{+}=0
$$

when the stray field is absent. The tunneling splitting at points 1 and 3 are caused by the local stray field. These two points are consistent with WAHC's work. ${ }^{13}$ The second process is from $|-9 / 2,-9 / 2\rangle_{+}$to $|7 / 2,7 / 2\rangle_{+}$via an intermediate state $|-9 / 2,7 / 2\rangle_{+}$. The two energy-level crossings are from $|-9 / 2,-9 / 2\rangle_{+}$to $|-9 / 2,7 / 2\rangle_{+}$at point 2 , and from $|-9 / 2,7 / 2\rangle_{+}$to $|7 / 2,7 / 2\rangle_{+}$at point 4 . We take the parameters for $D$ and $E$ for a SMM $\mathrm{Mn}_{4}$, and find that the calculated resonant fields are $b_{2}=0.233 \mathrm{~T}$ and $b_{4}=0.861 \mathrm{~T}$. Since points 2 and 3 are very close, the resonant peaks are smeared to produce a broader one. According to WAHC's paper there are only four metastable states $| \pm 9 / 2, \pm 9 / 2\rangle_{+}$in a dimer. All other excited states should relax to the metastable states in a very short time. Rigorously speaking, to realize the decay, one has to include a spin-phonon coupling in the Hamiltonian $^{21}$ even though the role of the spin-phonon coupling is not very clear since the spin-lattice relaxation becomes extremely long in low temperature. ${ }^{22}$ However, it was shown that such a spontaneous decay is necessary for understanding the hysteresis loop on $\mathrm{SMM} \mathrm{Mn} \mathrm{Mn}_{12} \cdot{ }^{21}$ Thus after the transition from $|-9 / 2,-9 / 2\rangle_{+}$to $|-9 / 2,7 / 2\rangle_{+}$at point 2 all particles decays from $|-9 / 2,7 / 2\rangle_{+}$to the metastable state $|-9 / 2,7 / 2\rangle_{+}$and no particles can reach the resonant point 4. Another resonant point is from $|-9 / 2,9 / 2\rangle_{+}$to $|7 / 2,9 / 2\rangle_{+}$at $b_{5}=0.943 \mathrm{~T}$. The tunneling from $|-9 / 2,-9 / 2\rangle_{+}$to $|-9 / 2,5 / 2\rangle_{+}$belongs to another triangle process and the splitting which is caused by the stray field is much smaller than those at points 2 and 4. Most particles tunnel into the other two states before reaching the point. On the other hand we anticipate that the weak coupling between two SMMs does not affect the intrinsic properties of a SMM in the dimer by much. It is worth pointing out that the coupling $\mathrm{J}$ can also drive the tunneling splitting between some states such as $|-9 / 2,+7 / 2\rangle_{+}$and $|9 / 2,7 / 2\rangle_{+}$. However, these tunnelings do not contribute significantly to what was observed by WAHC. ${ }^{13}$ We do not discuss them here.

After determining the positions of the resonant points and model parameters we are in a position to calculate the tunneling splitting, which determines the transition rate in the Landau-Zener model. The exact diagonalization method is applied to calculate the energy eigenvalues at different external field. The sampling average is taken for the local stray field. For each sampling we calculate the energy levels as in Fig. 1 and find the energy splitting $\Delta_{n}$ at each resonant point. More than 1000 samplings are taken to calculate the averaging tunneling splitting $\sqrt{\left\langle\Delta_{n}^{2}\right\rangle}$ for each distribution width $\sigma$. 
TABLE I. The calculated tunneling splitting $\sqrt{\left\langle\Delta^{2}\right\rangle}$ in units of $10^{-5} \mathrm{~K}$ at different resonant points with different distribution widths $\sigma$ of the stray field $\mathbf{h}$ using the exact diagonalization method. ( $D=0.762 \mathrm{~K}, E=0.0317 \mathrm{~K}$. $)$

\begin{tabular}{cccccc}
\hline \hline$\sigma / \mathrm{T}$ & 1 & 2 & 3 & 4 & 5 \\
\hline 0.000 & $<10^{-7}$ & 2.21907 & $<10^{-7}$ & 2.81552 & 1.52671 \\
0.010 & 0.01960 & 2.19227 & 0.01960 & 2.83557 & 1.59807 \\
0.020 & 0.03207 & 2.19487 & 0.03207 & 2.84268 & 1.59723 \\
0.035 & 0.04687 & 2.20155 & 0.04687 & 2.87249 & 1.61907 \\
0.050 & 0.06264 & 2.78338 & 0.06264 & 2.98306 & 1.68816 \\
\hline \hline
\end{tabular}

The calculated tunneling splitting are listed in Table I. We find that the tunneling splitting increases with the distribution width $\sigma$.

The derivatives of the hysteresis loops at different sweeping rates in Fig. 4 of WAHC's paper ${ }^{13}$ indicate that the peak heights in the derivatives depend on the sweeping rate. The height of the first peak decreases with the increasing rate, while oppositely the height of the second peak increases. This phenomenon can be understood qualitatively in the modified Landau-Zener model. In principle, the time evolution of the spin system can be reached by solving the 100 $[=(2 S+1)(2 S+1)]$ coupled time-dependent Schrödinger equation. Since the tunneling splitting is very small, the coupled equations near the two resonant states can be reduced to an effective two-level system with the Hamiltonian

$$
H_{e f f}=\left[\begin{array}{cc}
\left(m_{1},+m_{2}\right)\left(c_{b} t+h_{z}\right) & \Delta(\mathbf{h}) / 2 \\
\Delta(\mathbf{h}) / 2 & \left(m_{1}+m_{2}\right)\left(c_{b} t+h_{z}\right)
\end{array}\right] .
$$

The tunneling splitting $\Delta(\mathbf{h})$ between two states $\left|m_{1}, m_{2}\right\rangle_{+}$ and $\left|m_{1^{\prime}}, m_{2^{\prime}}\right\rangle_{+}$can be obtained by diagonalizing the Hamiltonian with a specific field $\mathbf{h}$. The state evolves with time,

$$
\Phi_{e f f}(t)=\mathbf{T} \exp \left[-\frac{i}{\hbar} \int_{-\infty}^{t} H_{e f f}(t) d t\right] \Phi_{e f f}(t=-\infty),
$$

where $\mathbf{T}$ is the time-ordered operator and the magnetization varying with time is given by $M(t)=\left\langle\Phi_{e f f}\left|\mathbf{S}_{1}^{z}+\mathbf{S}_{2}^{z}\right| \Phi_{\text {eff }}\right\rangle$. The average over the stray field $\mathbf{h}$ is taken for

$$
\langle d M(t) / d t\rangle=\int d \mathbf{h} P(\mathbf{h}) d M(t) / d t .
$$

Physically, with the local stray field, the Landau-Zener transition formula is given by

$$
P_{L Z}=1-\left\langle\exp \left[-\pi \Delta_{m m^{\prime}}^{2} / \nu_{m m^{\prime}}\right]\right\rangle \approx \pi\left\langle\Delta_{m m^{\prime}}^{2}\right\rangle / \nu_{m m^{\prime}},
$$

where

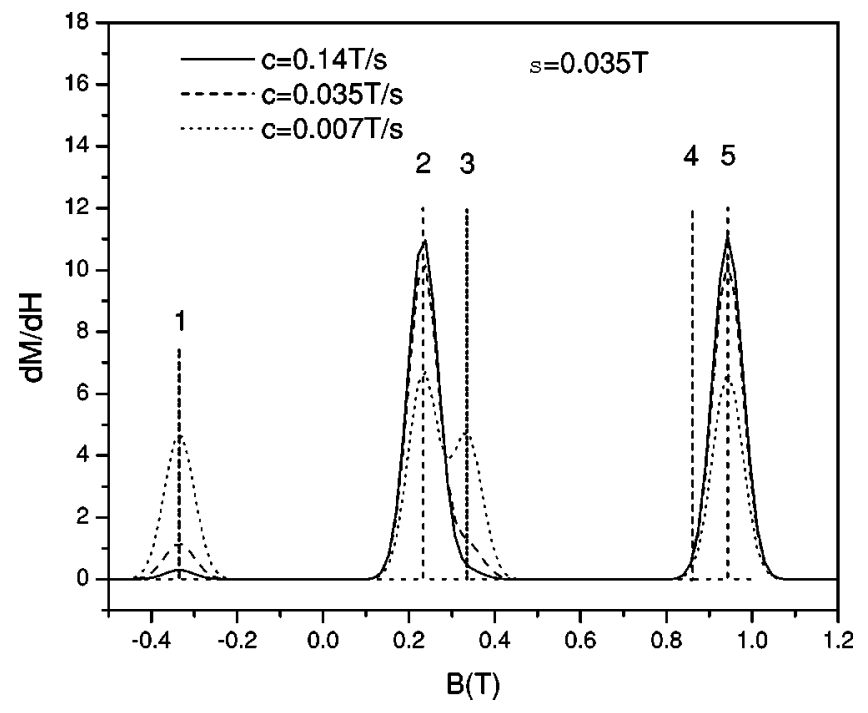

FIG. 3. Calculated derivative of the hysteresis loop at different sweeping fields based on the modified Landau-Zener model with the parameters $D=0.762 \mathrm{~K}, E=0.0317 \mathrm{~K}$, and $J=0.1 \mathrm{~K}$.

$$
\nu_{m m^{\prime}}=2 g \mu_{B} \hbar\left|\sum_{i=1,2}\left(m_{i^{\prime}}-m_{i}\right)\right| d B / d t .
$$

The rate is proportional to the reverse of the sweeping rate $c=d B / d t$, approximately. The larger the sweeping rate, the less particles tunnel to the new state. The step height is related to the transition rate by $\Delta M=P_{L Z} \Sigma_{i}\left(m_{i}-m_{i}^{\prime}\right)$. The presence of the local stray field will smear the "jump" of the magnetization around the resonant point. ${ }^{19}$ At a field $b$ around the resonant point, the variation of the magnetization due to quantum tunneling can be approximately given by $M(b) \simeq \Delta M \int_{-\infty}^{b} d \mathbf{h}_{z} P\left(\mathbf{h}_{z}\right)$, which leads to the derivative of the hysteresis loop around the resonant point, $d M / d b$ $\simeq \Delta M P(b)$. The calculated results are plotted in Fig. 3 . The first peak decreasing with the increasing rate is consistent with the Landau-Zener transition formula since $\mathrm{P}_{L Z}$ decreases with the sweeping rate. Oppositely, the second peak increases with the increasing rate, which seems to be inconsistent with $\mathrm{P}_{L Z}$. The magnetization change in the tunneling process is determined by two factors: one is the tunneling probability and another is the initial magnetization or number of particles before the tunneling. The peak height is determined by the particles' weight in the state as well as the transition rates. The less the particles transition from $|-9 / 2,-9 / 2\rangle_{+}$to $|-9 / 2,9 / 2\rangle_{+}$at point 1 the more the particles reach point 2 from $|-9 / 2,-9 / 2\rangle_{+}$to $|-9 / 2,7 / 2\rangle_{+}$. Although the transition rate is proportional to the reverse of the sweeping rate the second peak height increases as more particles reach at point 2 before the tunneling occurs for a larger sweeping rate. The competition of these two mechanisms determines the sweeping rate effect of the peak height. Comparing with Fig. 4 in WAHC's paper, ${ }^{13}$ we find that the numerical results based a Landau-Zener model are consistent with the experimental observation, and essentially reflect the sweeping rate effect on the peak height of derivatives of hysteresis loops. 
In conclusion, we studied the coupling effect of two truly identical particles, and analyze the quantum tunneling of magnetization in the supramolecular dimer of two $\mathrm{Mn}_{4} \mathrm{~s}$. The exchange coupling between two SMMs provides a biased field to expel the tunneling to two new resonant points via an intermediate state, and direct tunneling is prohibited. A stray field is introduced to explain the quantum splitting. Finally we point out that the sweeping rate effect in the derivatives of hysteresis loops can be explained quantitatively in the modified Landau-Zener model.

This work was supported by the Research Grant Committee of Hong Kong and a CRCG grant from the University of Hong Kong.
${ }^{1}$ D. D. Awschalom and D. P. Di Vincenzo Phys. Today 48 (4), 43 (1995).

${ }^{2}$ L. Thomas, F. Fionti, R. Ballou, D. Gatteschi, R. Sessoli, and B. Barbara, Nature (London) 383, 145 (1996).

${ }^{3}$ P. C. E. Stamp, Nature (London) 383, 125 (1996).

${ }^{4}$ E. M. Chudnovsky, Science 274, 938 (1996).

${ }^{5}$ R. Sessoli, D. Gatteschi, A. Caneschi, and M. A. Novak, Nature (London) 365, 141 (1993).

${ }^{6}$ W. Wernsdorfer and R. Sessoli, Science 284, 133 (1999).

${ }^{7}$ Macroscopic Quantum Coherence and Quantum Computing, edited by D. V. Averin, B. Ruggiero, and P. Silvestrini (Kluwer Academic/Plenum, New York, 2001).

${ }^{8}$ W. Wernsdorfer, Adv. Chem. Phys. 118, 99 (2001).

${ }^{9}$ S. Wang, H. L. Tsai, E. Libby, K. Folting, W. E. Streib, D. N. Hendrickson, and G. Christou, Inorg. Chem. 35, 7578 (1996).

${ }^{10}$ S. M. Aubin, N. R. Diley, M. W. Wemple, M. B. Maple, G. Christou, and D. N. Hendrickson, J. Am. Chem. Soc. 120, 839 (1998).

${ }^{11}$ H. Andres, R. Basler, H. U. Gudel, G. Aromi, G. Christou, H. Buttner, and B. Ruffle, J. Am. Chem. Soc. 122, 12469 (2000).

${ }^{12}$ W. Wernsdorfer, S. Bhaduri, C. Boskovic, G. Christou, and D. N.
Hendrickson, Phys. Rev. B 65, 180403 (2002).

${ }^{13}$ W. Wernsdorfer, N. Allaga-Alcalde, D. N. Hendrickson, and G. Christou, Nature (London) 416, 406 (2002).

${ }^{14}$ M. N. Leuenberger and D. Loss, Nature (London) 410, 789 (2001).

${ }^{15}$ B. Zhou, R. B. Tao, S. Q. Shen, and J. Q. Liang, Phys. Rev. A 66, 010301(R) (2002).

${ }^{16}$ J. L. van Hemmen and S. Suto, Europhys. Lett. 1, 481 (1986).

${ }^{17}$ D. Loss, D. P. DiVincenzo, and G. Grinstein, Phys. Rev. Lett. 69, 3232 (1992).

${ }^{18}$ Jan von Delft and C. L. Henley, Phys. Rev. Lett. 69, 3236 (1992).

${ }^{19}$ Z. D. Chen, J. Q. Liang, and S. Q. Shen, Phys. Rev. B 66, 092401 (2002).

${ }^{20}$ As there exists higher-order unknown transverse terms in the model Hamiltonian, the tunnel splitting calculated in this paper is comparable with the experimental data qualitatively.

${ }^{21}$ K. Saito, S. Miyashita, and H. de Raedat, Phys. Rev. B 60, 14553 (1999).

${ }^{22}$ M. Ueda, S. Maegawa, and S. Kitagawa, Phys. Rev. B 66, 073309 (2002). 